\title{
To compare the clinical effectiveness of azithromycin vs doxycycline on the first follow-up visit of acne vulgaris patients
}

\begin{abstract}
Acne Vulgaris is a chronic inflammatory disorder of pilosebaceous unit which frequently affects the cosmetically important areas of the body like face, back, chest, neck, and upper arms. It is a multifactorial disease and its pathophysiology centres on the interplay of increased sebum production, follicular hyperkeratinisation, colonization with Propionibacterium Acnes and inflammation. There are many systemic modalities for the treatment of acne among which Azithromycin and Doxycycline are the commonly prescribed ones. With an aim to manage patients effectively a case-control study was conducted on 70 patients to compare the clinical effectiveness of both the drugs for the early symptomatic relief of moderate to severe inflammatory acne.
\end{abstract}

Method This study was conducted at the outdoor of dermatology department at Punjab Rangers Teaching Hospital over a period of three months starting from August 2019 till the end of October. A total of 70 acne patients compatible with the inclusion criteria were enrolled and divided into two equal groups of cases and controls. The cases were prescribed Azithromycin 500mg once a day for first two weeks followed by $250 \mathrm{mg}$ once a day for the next two weeks, while the controls were prescribed Doxycycline $100 \mathrm{mg}$ once a day. Both groups were treated for a period of four weeks and were assessed based on new lesion count and the settlement of older lesions to compare the clinical effectiveness of each drug.

Results Based on the clinical assessment at the first follow-up after 4 weeks, it was observed that in the Azithromycin group 21 patients $(60 \%)$ showed a good response with (more than 50\%) clearance of the inflammatory pustules and nodules, $9(26 \%)$ patients showed a moderate response between $30-50 \%$ and $5(14 \%)$ patients showed a mild response between $20-30 \%$. In Group B, 12 (34\%) patients showed moderate response between 30$50 \%, 12(34 \%)$ patients showed mild response between $20-30 \%$, while the remaining 11 $(32 \%)$ patients were placed in the no response category with less than $20 \%$ improvement. Overall Azithromycin showed significantly better response, p-value \&1t; 0.001 than other group, $\mathrm{p}$-value $=0.014$.

Conclusion It was observed that Azithromycin has an earlier onset of action in the treatment of inflammatory acne as compared to Doxycycline. So, for patients where prompt results are desired Azithromycin has proven to be more effective as compared to Doxycycline.
Volume 4 Issue 3 - 2020

\author{
Nadia Sultan, Merub Dar, Zoya Rehman, \\ Ghazala Butt, Saira Omer, Saima Rehman, \\ Asad Ali Chaudhry, Fatima Mehak \\ Department of dermatology, Mayo Hospital KEMU, Pakistan
}

Correspondence: Ghazala Butt, Department of dermatology, Mayo Hospital, King Edward Medical University, Pakistan, Email ghazalakashiri@gmail.com

Received: June 09, 2020 | Published: June 30, 2020

Keywords: acne, azithromycin, doxycycline

\section{Introduction}

Acne is an inflammatory disease of the pilosebaceous unit appearing on the face, neck, chest \& back resulting from androgen-induced increased sebum production, altered keratinization, inflammation, and bacterial colonisation of hair follicles by Propionibacterium acnes. Multiple factors contribute to the pathogenesis of this disease among which early colonisation with $\mathrm{P}$ acnes and family history might have important roles, however it remains unclear what exactly triggers the disease and how treatment affects its course. ${ }^{1,2}$

The key pathogenic factors that have been known for years include follicular epithelial hyperproliferation resulting in plugging of the follicles, excess sebum production \& the presence and activity of Propionibacterium acnes. ${ }^{3}$ Nearly $100 \%$ of individuals develop acne at some stage of their life with variable severity. Acne has a deep impact on the patient's quality of life, with small, non inflamed acne lesions not causing more than a slight nuisance but, in individuals with more severe inflammatory nodular acne, the pain, social embarrassment \& both physical and psychological scarring can be life altering. The most common candidates for acne are adolescents, where the onset of this disease leaves a deep emotional and psychological upset which creates developing issues such as dissatisfaction with appearance, lack of selfconfidence and social anxiety with the opposite gender and peers. ${ }^{4}$ Fortunately, our therapeutic armamentarium has greatly expanded over the last few years due to progression in the understanding of the pathogenesis of this disease. ${ }^{3}$ Acne can be classified into four grades:

- GRADE I comedones, occasional papules.

- GRADE II Papules, comedones, few pustules.

- GRADE III predominant pustules, nodules, abscesses.

- GRADE IV mainly cysts, abscesses, widespread scarring. 
Grade I and II are usually treated as mild acne whereas grade III and IV are treated as moderate to severe acne. Besides the widespread use of conservative management protocols like controlling dietary factors, face washing, topical antibacterial and comedolytics, oral antibiotics remain the first line of treatment for acne patients who are afflicted with physical and psychological side-effects of the severe form of this disease. ${ }^{4}$ Mild acne can be effectively treated with only topical therapy, however, in moderate to severe acne only topical medication is insufficient, therefore systemic antibiotics have long been used for its treatment. There are few limitations to the use of systemic antibiotic therapy for the treatment of acne vulgaris, as they could only be prescribed for the briefest possible time frame, to reduce the risk of developing resistance and their use should be assessed after three to four months, to screen for the side effects. Data is limited regarding the use of antibiotics that are not tetracyclines or macrolide; therefore, their use should be avoided. ${ }^{5}$

The most commonly used antibiotics are Tetracyclines and Macrolides which have shown promising results in the past. Azithromycin is an azalide of macrolide group of antibiotics, derived from erythromycin by insertion of a nitrogen atom in the macrolide ring. This addition has an important advantage over erythromycin including greater tissue penetration and extended half-life. ${ }^{6}$ It is primarily a bacteriostatic drug, but in higher concentration can be bactericidal. $^{7}$ Doxycycline is a semisynthetic, second generation, broad-spectrum tetracycline derived from oxytetracycline. Currently, it is widely used among others, in the treatment of acne and skin infections, due to its antibacterial and anti-inflammatory effects. ${ }^{8,9}$ There is a consensus that oral antibiotic therapy should not exceed 3 to 4 months and that a minimum duration of six weeks is commonly required to see clinical improvement. ${ }^{12}$ Thus keeping this in mind, we planned to conduct a study and select a drug which could show a significant response in an early time span to inculcate a sense of satisfaction to the patients, thus ensuring their compliance.

\section{Material and method}

A prospective, comparative study conducted at the outdoor of dermatology department of Punjab Rangers Teaching Hospital, Lahore over a period of three months starting from August 2019 till the end of October. A total of 70 acne patients who were compatible with the inclusion criteria were enrolled in the study. The data about age, gender, site of lesion, duration of disease \& grade of acne was kept on record. Acne was graded as mild, moderate and severe according to the grading already mentioned, ${ }^{4}$ Only moderate to severe inflammatory acne patients were enrolled in this study. An informed consent was taken from the patients to ensure their compliance thus avoiding bias. Out of total 70 patients, two equal groups were formed, group A and group B. Thirty-five patients were allocated in either group.

\section{Inclusion criteria}

Both males and females between 15-35 years of age, with moderate to severe acne were enrolled in this study.

\section{Exclusion criteria}

The following patients were excluded from the study:

- Patients under 15 years

- Known Gastritis

- Known hypersensitivity to the drugs being tested

- Pregnancy

Group A patients were prescribed Azithromycin 500mg once a day dosage for two weeks followed by $250 \mathrm{mg}$ once a day dosage for the next two weeks. The group B patients were prescribed doxycycline $100 \mathrm{mg}$ once a day dosage for four weeks. Both groups were followed after a period of 4 weeks. On the first follow-up both subjective and objective clinical evaluation was made based on the degree of settlement of the inflammatory pustules \& nodules \& the count of new eruptions.

From group A (Azithromycin), out of 25 patients of grade III acne, 16 patients had significant improvement with more than $50 \%$ settlement of the inflammatory nodules and pustules, and remaining 9 patients had between $30-50 \%$ improvement. Erythema had started to settle down over most of the inflammatory lesions. Out of 10 patients of severe acne, 5 patients showed very good response with $50 \%$ reduction in size of cyst and nodule.. However, the remaining 5 patients only $20 \%$ response was noted (Figure 1-3).

\section{Azithromycin (Group A)}

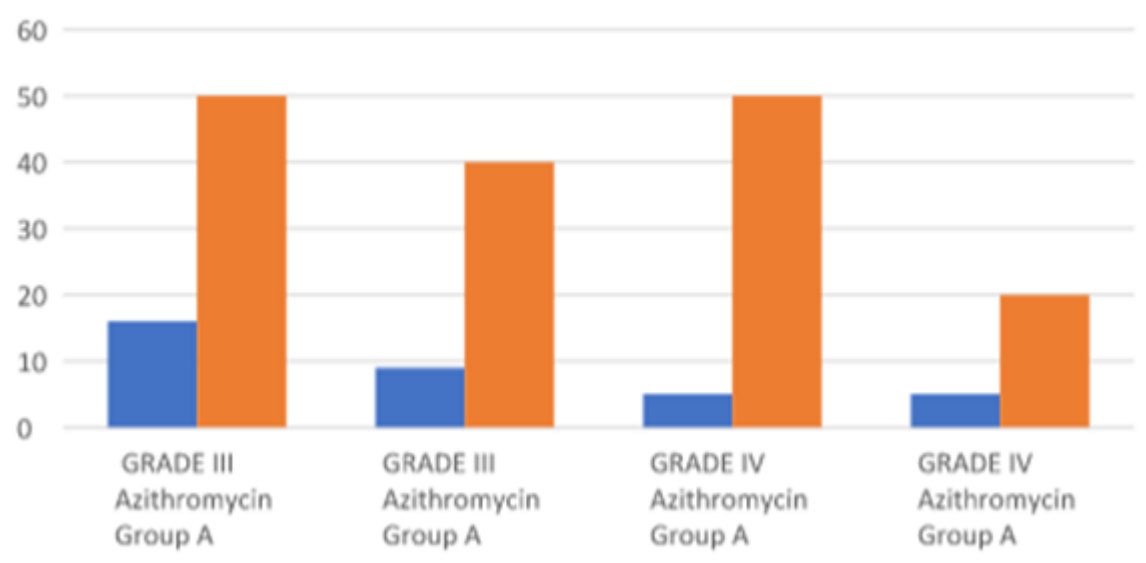

NO. OF PATIENTS DERCENTAGE(\%)

Figure I Group A (Azithromycin). 


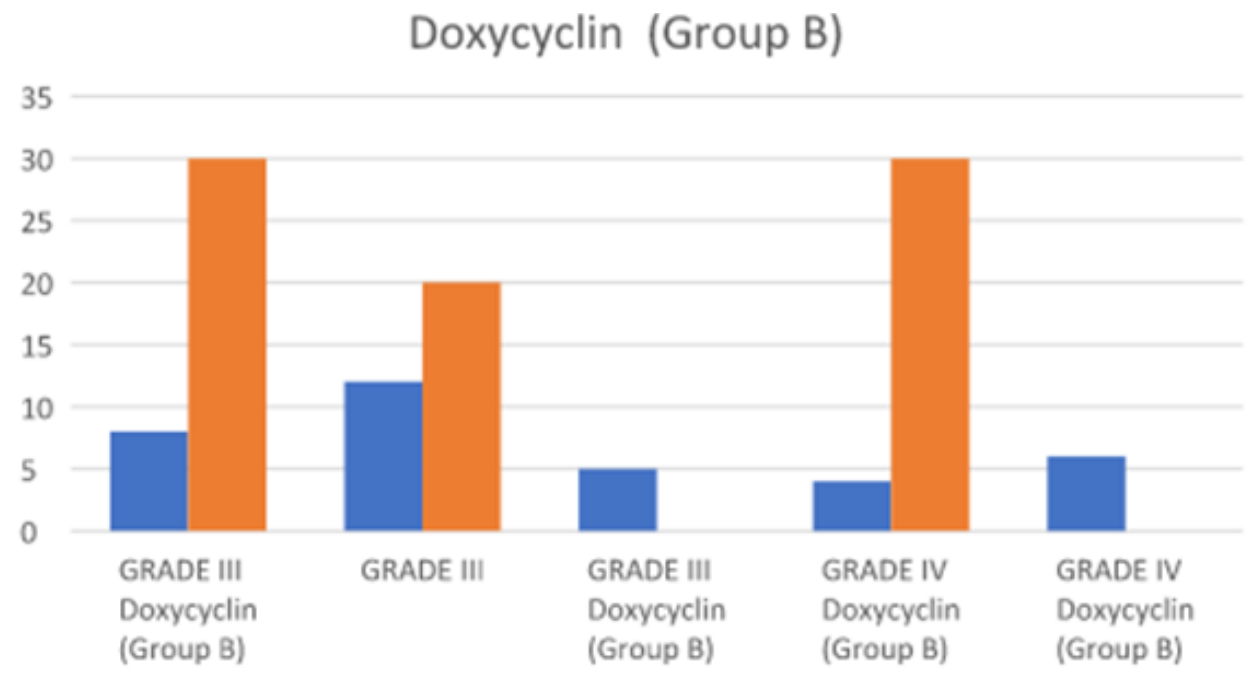

NO. OF PATIENTS DERCENTAGE(\%)

Figure 2 Group B (Doxycycline).

\section{Effects of Azithromycin and Doxycyclin}

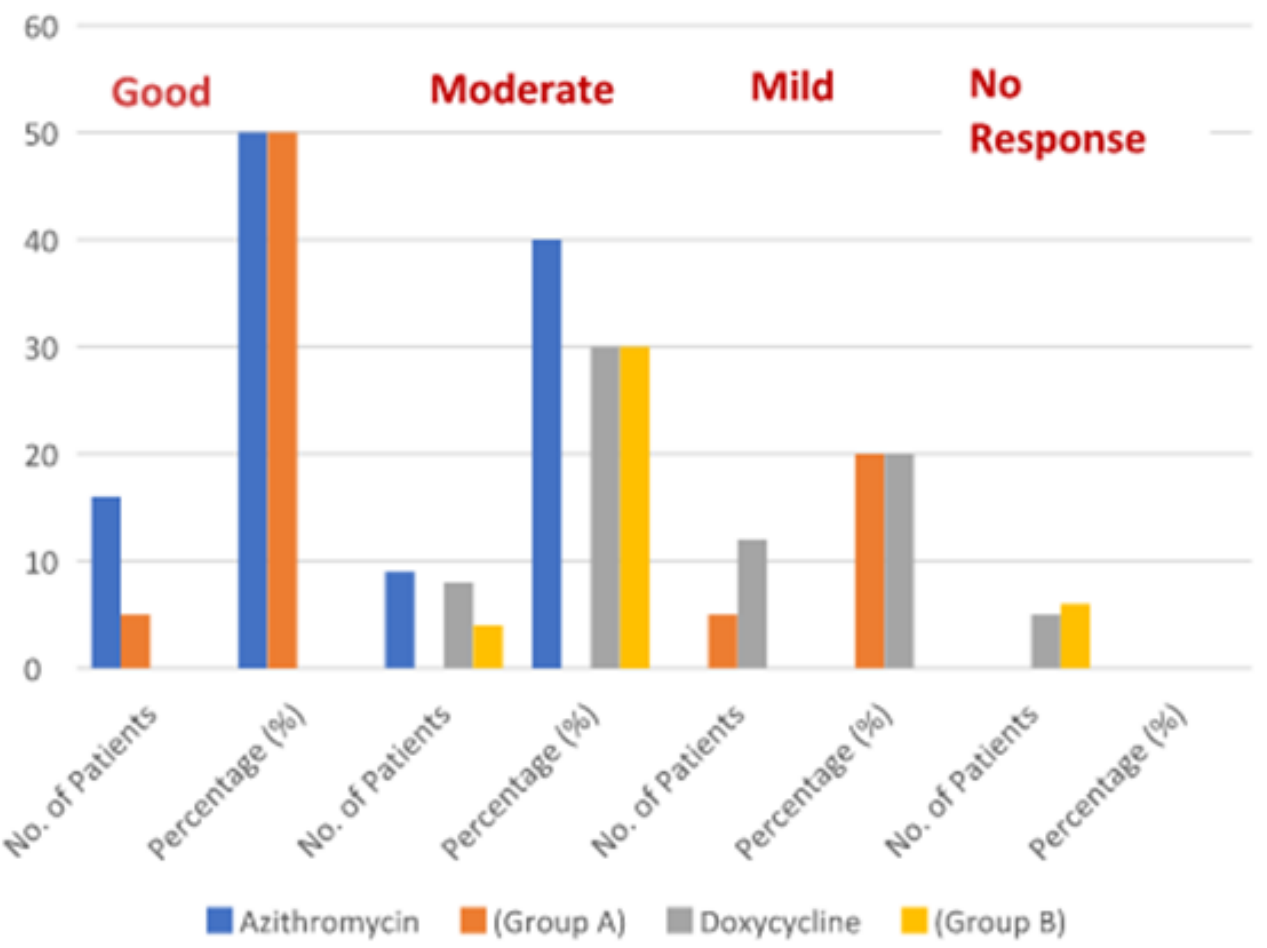

Figure 3 Effects of Azithromycin \& Doxycycline.

From group B (Doxycycline) out of 25 patients of grade III acne only 8 patients had shown $30 \%$ improvement, while out of remaining 17 patients, 12 patients showed only $20 \%$ improvement, while remaining 5 patients had no significant change. Out of 10 patients of grade IV acne only 4 patients showed $30 \%$ improvement with reduction in erythema and slight reduction in size of nodule with no significant effect on cyst and the response of remaining 6 patients was insignificant.

\section{Results}

Based on the clinical assessment on the first follow-up after 4 weeks, it was observed that in the Azithromycin group 21 patients $(60 \%)$ showed a good response with (more than 50\%) clearance of the inflammatory pustules and nodules, $9(26 \%)$ patients showed a moderate response between $30-50 \%$ and $5(14 \%)$ patients showed a mild response between 20-30\%. In Group B, 12 (34\%) patients 
showed moderate response between $30-50 \%, 12$ (34\%) patients showed a mild response between $20-30 \%$, while the remaining 11 $(32 \%)$ patients were placed in the no response category with less than $20 \%$ improvement. (Table 1-4)

Table I Division of patient's genger

\begin{tabular}{llll}
\hline Group/Gender & Male & Female & Total \\
\hline Group A & 14 & 21 & 35 \\
Group B & 16 & 19 & 35 \\
Total & 30 & 40 & 70 \\
\hline
\end{tabular}

Table 2 Age group between 15 to 35 (mean average of both groups)

\begin{tabular}{lll}
\hline Age-Limits & Group A & Group B \\
\hline $15-20$ & 17 & 15 \\
$21-25$ & 10 & 12 \\
$26-30$ & 4 & 7 \\
$31-35$ & 4 & 1 \\
Total & 35 & 35 \\
Average & $22.2 \pm 3.24$ & $22.34 \pm 4.27$
\end{tabular}

Table 3 Grades of acne in group A \& B

\begin{tabular}{lllll}
\hline Groups & Grades & Male & Female & Total \\
\hline Group A & Grade III & 10 & 15 & 25 \\
& Grade IV & 4 & 6 & 10 \\
Group B & Grade III & II & 14 & 25 \\
& Grade IV & 5 & 5 & 10 \\
& Total & & & \\
\hline
\end{tabular}

Table 4 Response to treatment with \%age

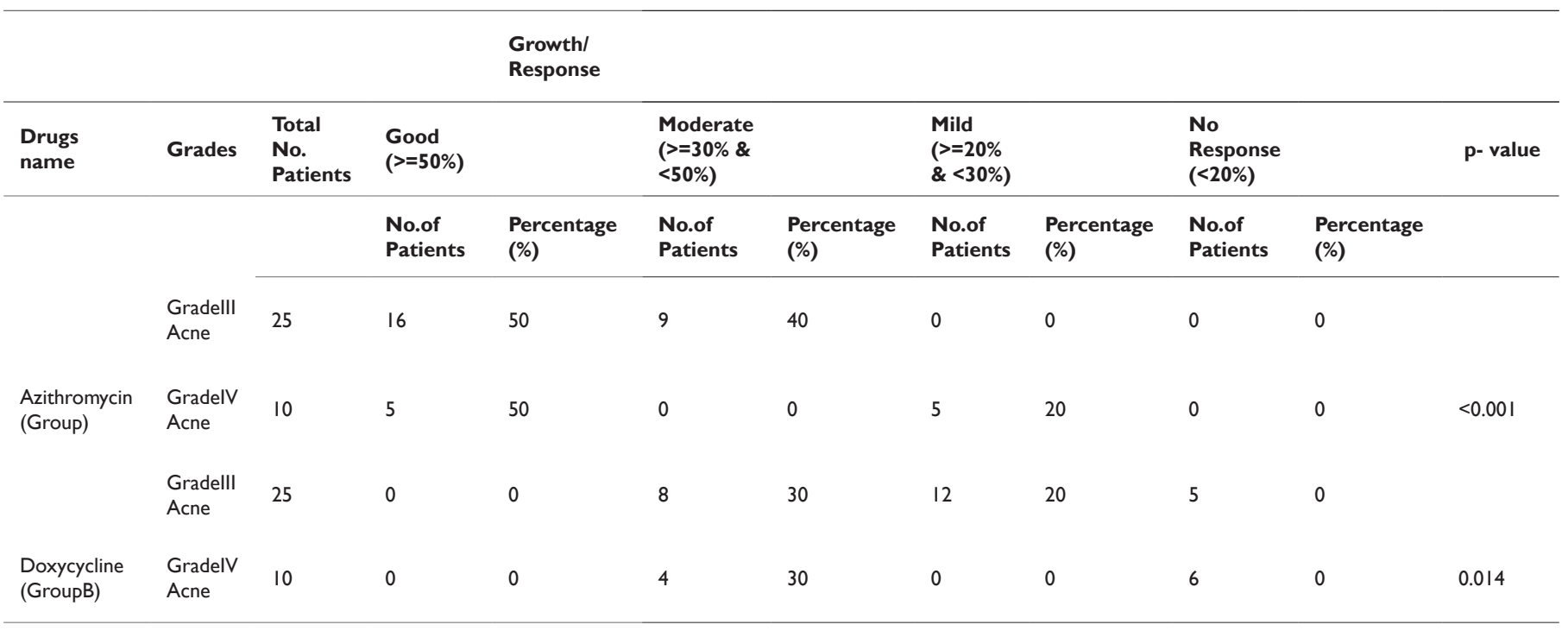

\section{Discussion}

Acne vulgaris is a skin disease that has a deep psychological impact on its patients. Studies have been conducted all over the world over the multiple treatment modalities for this disease with variable outcome. So far, the most common systemic agents advocated for use in moderate to severe inflammatory acne stand the systemic antibiotics, amongst which Azithromycin \& Doxycycline are the most frequently tested \&; prescribed ones. Similar studies have been conducted in the past to analyse the difference between the clinical effectiveness of both drugs $\&$ the results are more or less comparative to the results of our study. A comparative study between Azithromycin \& Doxycycline with pulse dosing of Azithromycin 500mg for a period of 3months \& 100mg Doxycycline once daily, showed better results with Azithromycin (77.26\%) as compared to Doxycycline $(63.74 \%)$ but the outcome was measured after three months time, the dose of Azithromycin was in pulses. ${ }^{10} \mathrm{Kim}$ JE included the results of six studies assessing 906 patients of moderate to severe acne vulgaris, on the comparative efficacy of the same drugs and showed equivalent results. Azithromycin pulse therapy was proven equivalent to doxycycline $100 \mathrm{mg}$ daily dose at 12 weeks, for the treatment of moderate to severe acne vulgaris. ${ }^{11}$

Another study carried out in Italy by Bardazzi, on the clinical effectiveness of Azithromycin which enrolled fifty-two adolescent patients of moderate to severe papulopustular acne vulgaris given Azithromycin, 500mg thrice weekly for 8 weeks, quoted similar results to our study with $90.4 \%$ patients showing remarkable 
improvement within the first 4 weeks, with a significant reduction in their inflammatory papulo-pustular lesions (23 showed "moderate" reduction and 24 "good-excellent"). Maximum clearance was observed at 8 weeks. This study proved that this drug appears to be a safe and effective treatment for acne vulgaris in adolescents, with excellent patient compliance. ${ }^{12}$ One more study reporting similar results like our study by Sanjeev Sharma on 200 patients dosing Azithromycin 500mg thrice weekly also supports that this drug has greatest advantage over other systemic antibiotics in acne. The primary focus of this openlabel non-comparative therapeutic study was to assess the efficacy of $500 \mathrm{mg}$ of azithromycin thrice weekly (once on every other day) for 8 weeks in the treatment of Acne vulgaris in TMUpatients. ${ }^{13}$

Although literature surfing reveals quite a significant data on the comparative efficacy of the Azithromycin versus Doxycyclin, however Azithromycin has mostly been tested in a pulse dosage, the response assessed after a minimum time period of two to three months. These treatment regimens though prove effective, however the clinical response is somewhat delayed which hers patients compliance to the treatment, at the same time weakening his/her confidence in the therapy as well as the treating dermatologist. The rationale of the present study was to counter a new challenge in using a different dosing regimen of Azithromycinthen assess the clinical outcome of the treatment at the first follow up visit of our patients which was set to be after 4 weeks period, to evaluate that which of the two drugs was more effective in causing an earlier response in the settlement of moderate to severe inflammatory acne, which proved Azithromycin to be superior in efficacy to Doxycyclin. The present study thus supports the use of Azithromycin in a daily dose for a limited time giving

promising results which can later on be tapered down to pulse dosing after settlement of the initial flare of the inflammatory acne. Therefore, for those patients where an expeditious response is desired, Azithromycin outweighs Doxycyclin in proving an eminent response in a short span of time thus giving the patient a satisfying outcome, as a late onset of response creates a state of dissatisfaction to the patients and increases the possibility of non-compliance to the treatment \&; instilling further psychological distress.

\section{Conclusion}

With regards to milder side effects and their lower incidence and its anti-inflammatory properties, Azithromycin is a good choice for the treatment of moderate to severe inflammatory acne as compared to other commonly prescribed oral antibiotics. Due to its effectiveness and safe use during pregnancy, this drug is a promising treatment for lactating and pregnant women suffering from acne vulgaris. Despite, the on-going discussion about the developing resistance to azithromycin in various infections, no resistance has yet been reported regarding its use in acne vulgaris. Therefore, we encourage the use of Azithromycin to attain optimal results in moderate inflammatory acne.

\section{Conflicts of interest}

The author declares that there is no conflicts of interest.

\section{Acknowledgments}

None.

\section{Funding}

None.

\section{References}

1. Amatya, Chaudhary K, Khan DK. A Comparative Study of Azithromycin Versus Doxycycline in the Treatment of Acne Vulgaris. Nepal Journal of Dermatology, Venereology \& Leprology. 2012;10(1).

2. Williams HC, Dellavalle RP, Garner S. Acne vulgaris. The Lancet. 2012;379(9813):361-72.

3. Harper JC. An update on the pathogenesis and management of acne vulgaris. Journal of the American Academy of Dermatology. 2004;51(1):36-38.

4. Kardeh S, Saki N, Jowkar F, et al. Efficacy of Azithromycin in Treatment of Acne Vulgaris: A Mini Review. World J Plast Surg. 2019;8(2):127-134.

5. Croke L. Acne Vulgaris: Treatment Guidelines from the AAD. 2020.

6. Kim M, Welch T. Update on azithromycin and cardiac side effects metastatic pulmonary calcification. Southwest Respir Crit Care Chron. 2014;2(5):48-51.

7. Sardesai V, Deka Y. Comparison of efficacy of oral azithromycin with oral minocycline in the treatment of acne vulgaris. Clinical Dermatology Review. 2017;1(2):37-40.

8. Rok J, Karkoszka M, Rzepka Z, et al. Cytotoxic and proapoptotic effect of doxycycline - An in vitro study on the human skin melanoma cells. Toxicology in Vitro. 2020;65:104790.

9. Doxycycline Drug Bank. 2020.

10. Singhi M K, Ghiya B C, Dhabhai R K. Comparison of oral azithromycin pulse with daily doxycycline in the treatment of acne vulgaris . Indian $J$ Dermatol Venereol Leprol. 69(4):274-276.

11. Kim JE, Park AY, Lee SY, et al. Comparison of the Efficacy of Azithromycin Versus Doxycycline in Acne Vulgaris: A Meta-Analysis of Randomized Controlled Trials. Ann Dermatol. 2018:30(4):417-426.

12. Bardazzi, Federico, Francesco, Parente, Tabanelli, Michela, et al Azithromycin: A new therapeutical strategy for acne in adolescents. Dermatol Online J. 2007;13(4):4.

13. Oon HH, Wong SN, Aw DCW, et al. Acne Management Guidelines by the Dermatological Society of Singapore. The Journal of Clinical and Aesthetic Dermatology. 2019;12(7):34-50. 